FORMATION Formation emploi

Revue française de sciences sociales

127 | juillet-septembre 2014

Pêle mêle

\title{
Aides à domicile : la formation améliore-t-elle l'emploi ?
}

Social Life Auxiliary : does training improve the quality of employment?

Sozialpfleger : Verbessert eine Ausbildung die Beschäftigungsqualität?

Ayudas a domicilio : la formación ¿mejora el empleo?

\section{Loïc Trabut}

\section{(2) OpenEdition}

Journals

\section{Édition électronique}

URL : http://journals.openedition.org/formationemploi/4254

DOI : 10.4000/formationemploi.4254

ISSN : 2107-0946

\section{Éditeur}

La Documentation française

\section{Édition imprimée}

Date de publication : 30 octobre 2014

Pagination : 71-90

ISSN : 0759-6340

\section{Référence électronique}

Loïc Trabut, « Aides à domicile : la formation améliore-t-elle l'emploi ? », Formation emploi [En ligne],

127 | juillet-septembre 2014, mis en ligne le 30 octobre 2016, consulté le 30 octobre 2020. URL

http://journals.openedition.org/formationemploi/4254; DOI : https://doi.org/10.4000/

formationemploi.4254 


\section{Aides à domicile : la formation améliore-t-elle l'emploi ?}

LOÏC TRABUT

Sociologue, chargé de recherche à l'Institut National d'Etudes Démographiques, associé au

Centre d'Études de l'Emploi.

Résumé

Aides à domicile : la formation améliore-t-elle l'emploi ?

Quel est l'effet de la mise en place du diplôme d'État d'Auxiliaire de vie sociale (DEAVS) sur la qualité de l'emploi du secteur de l'aide à domicile ainsi que sur les critères de recrutement ? Fondé sur une enquête ethno-statistique menée auprès d'une association d'aide à domicile, cet article montre que la valorisation symbolique et salariale se réalise au détriment des conditions d'emploi. La norme hétéronome de flexibilité, à l'origine de la mauvaise qualité d'emploi, est indépendante de la professionnalisation par la formation.

Mots clés : aide à domicile, certification, diplôme, auxiliaire de vie sociale, recrutement, validation des acquis

Abstract

Social Life Auxiliary: does training improve the quality of employment?

What is the effect of the State Diploma for Social Life Auxiliary (Diplôme d'État d'Auxiliaire de Vie Sociale, DEAVS) on the quality of employment in the sector of elderly care as well as on the criteria for recruitment? Based on an ethno-statistical investigation conducted in an elderly care organisation, this paper shows that symbolic and wage valorization is achieved at the expense of employment conditions. The heteronomous standard of flexibility, causing the poor quality of jobs, is independent of professionalization through training.

Keywords : home help, certification, diploma, social life auxiliary, recruitment, validation of acquired skills Journal of Economic Literature : $\mathbf{J} 4 \mathbf{4}$

Traduction : Auteur 
En 2008, la Drees (Direction de la recherche, des études, de l'évaluation et des statistiques) dénombrait 515000 aides à domicile intervenant au domicile de personnes fragilisées, que ce soit en raison de leur âge, d'un handicap, d'une maladie ou de toute autre raison (Marquier, Hanon, 2012). Ces salariés intervenaient auprès des personnes nécessitant une aide pour réaliser les actes essentiels de la vie quotidienne, tels que se laver, manger, etc. Ces interventions sont principalement financées dans le cadre de prestations sociales. La plus importante est l'Allocation Personnalisée d'Autonomie (APA), qui s'élève, en 2008, à 3,2 milliards d'euros. Elle est versée aux personnes âgées de plus de 60 ans en perte d'autonomie. $92 \%$ des montants versés financent des heures d'aide à domicile.

Alors que le terme d'aide à domicile existe dans la loi depuis $1956^{1}$, aucun texte n'a réellement traité de la définition du métier jusqu'en 1970, à l'occasion de la rédaction des premières conventions collectives, dont aucune ne retient alors la même appellation. Néanmoins, le 29 mars 2002, un accord relatif à l'emploi et aux rémunérations, agréé par un arrêté du 31 janvier 2003, est signé. Il a pour vocation de définir les métiers, de créer des filières professionnelles et d'homogénéiser les différents statuts. Un processus d'unification est alors lancé pour doter ce secteur d'une convention collective unique (la convention collective de la branche de l'aide, de l'accompagnement, des soins et des services à domicile 2007), entrée en vigueur le $1^{\text {er }}$ janvier 2012, et d'un diplôme unique, le DEAVS, diplôme d'Etat d'Auxiliaire de vie sociale² (Moreau, 2003).

Stéphane Alvarez considère cet accord de branche comme la dernière étape du processus de professionnalisation du secteur : "Les anciennes aides ménagères et aides à domicile se voient offrir une possibilité de qualification à travers l'instauration du diplôme d'Etat d'Auxiliaire de vie sociale (DEAVS). Le changement de nom de l'aide à domicile en auxiliaire de vie correspond à une volonté de modifier la position sociale de ces "aides à domicile”. » (Alvarez, 2010, pp. 5-6). Ce diplôme de niveau V³ les place désormais au même niveau de formation et de rémunération que les aides-soignantes (Moreau, 2003). Ce consensus en faveur d'une professionnalisation permettrait d'offrir des perspectives plus incitatives aux jeunes générations. Toutefois, la formation n'est pas l'unique levier vers la professionnalisation de l'aide à domicile (Croff, Mauduit, 2003); cela suppose aussi des emplois plus proches de l'emploi moyen en termes de durée du travail, de revenus, et de perspectives d'évolution (Maruani, 2011).

1. Article 157, Chapitre V "Aide sociale aux personnes âgées du Code de la famille et de l'aide sociale ", publié au Journal Officiel de la République française, 28 janvier 1956.

2. 3519 diplômes validés en formation et 2676 par acquis de l'expérience en 2011 (Nahon, 2013).

3. Niveau de formation équivalent à celui du brevet d'études professionnelles (BEP) ou du certificat d'aptitude professionnelle (CAP). 


\section{Encadré 1 : Méthodologie}

Ce texte s'inscrit dans le prolongement d'un travail de thèse visant à appréhender les modalités de production de l'aide à domicile pour personnes âgées.

II s'appuie sur une enquête réalisée, de 2009 à 2010, sur l'ensemble des processus de production, depuis le recrutement des salariés, l'organisation du travail, jusqu'à la production du service dans une association d'aide à domicile fournissant des interventions aux particuliers sous différentes modalités. Nous traitons ici uniquement des salariés directement employés par l'association, c'est-à-dire travaillant sous une forme prestataire.

L'aide à domicile correspond à trois modes d'organisation du travail : Les modes prestataire ou mandataire dans le cas d'une aide intermédiée par une association ou une entreprise et l'emploi direct dans le cas d'une aide directement recrutée par la personne âgée. Dans le cas du mandataire, la responsabilité légale de l'employeur revient au bénéficiaire du service, dans le cas du prestataire, à l'organisme employeur qui vend ensuite la prestation.

En 2008, 72,6 \% des services étaient orientés vers les personnes « vulnérables » (a), plus de 122000 heures de prestations étaient fournies à 350 clients sur l'année et 55,7\% des heures vendues l'étaient dans le cadre de l'Allocation Personnalisée d'Autonomie (APA). L'allocation est destinée à couvrir en partie les dépenses de toute nature (principalement d'aide à domicile) afin d'accomplir des actes essentiels de la vie. L'APA à domicile est attribuée, sous certaines conditions, par les conseils généraux qui, par l'intermédiaire des équipes médicosociales, définissent des plans d'aide.

Ce mode de financement oblige l'association à suivre une prescription d'intervention fournie par le conseil général. Nous avons collecté ces plans d'aide $\left(^{*}\right)$ pour les 89 allocataires de I'APA. Les autres clients payent directement les prestations à l'association, majoritairement du ménage.

L'enquête s'est déroulée sur une période de dix mois, au cours de laquelle nous nous sommes déplacés sur le territoire d'intervention de l'association et dans l'association, de un à trois jours par semaine. Outre l'observation du fonctionnement de la structure et le recueil des données relatives aux ressources humaines, l'objectif était de réaliser des entretiens avec le personnel en charge de la gestion de la main-d'œuvre (5), et avec certains salariés intervenant à domicile (10).

Les données collectées nous permettent d'identifier les profils et caractéristiques sociales de 172 salariés ayant été recrutés de manière pérenne (b) depuis 1999 et toujours en emploi. L'échantillon de salariés sur lequel nous avons travaillé représente l'ensemble des salariés de l'association travaillant au moins en partie en mode prestataire (112/172) (c) et réalisant soit des services dits de " confort » correspondant à du ménage, soit de I' " aide à la personne », c'est-à-dire aide à la toilette, à l'habillage, aux déplacement, etc., selon les termes utilisés.

Le dossier administratif rempli pour chaque salarié permet de connaître ses caractéristiques. Lors du recrutement, chaque salarié, outre le CV qu'il fournit, remplit une fiche précisant son statut actuel, son parcours professionnel, ses formations, sa situation familiale, ses disponibilités ainsi que les publics avec lesquels il souhaite travailler. Cette procédure a changé au cours de l'enquête. L'externalisation d'une partie du recrutement vers la maison de l'emploi locale a contraint l'employeur à objectiver ses critères de sélection des candidats afin de les transmettre aux agents recruteurs qui reçoivent et filtrent les candidats pour l'association. 


\section{Encadré 1 (Suite)}

Afin de comprendre les effets du diplôme sur l'emploi, nous avons collecté, en 2009, sur deux semaines (d), l'ensemble des emplois du temps des salariés de l'association. Les 112 salariés travaillant au moins en partie en mode prestataire réalisent 3213 interventions planifiées sur deux semaines. En raison du départ d'une des cadres, responsable "qualité et recrutement », la collecte des données administratives n'est plus systématique depuis fin 2008 et les salariés récemment recrutés ne sont donc pas intégrés à cette analyse. II nous a été possible d'apparier uniquement 2496 interventions des emplois du temps des salariés et leurs caractéristiques de formation.

Notre analyse porte donc sur l'observation de près de $80 \%$ des emplois du temps des salariés en mode prestataire sur une période de deux semaines. Notre présence pendant dix mois au sein de l'association nous permet d'établir que cette observation est représentative du fonctionnement général de cette association. Dans la mesure où les conseils généraux sont libres de fixer les tarifs et l'organisation de la prise en charge financière dans un cadre légal, notre analyse ne peut être généralisée à la France entière de ce point de vue.

a) Pour le code pénal, il s'agit d'une personne dont la particulière vulnérabilité est due à son âge, à une maladie, à une infirmité, à une déficience physique ou psychique ou à un état de grossesse.

b) Les salariés recrutés uniquement pour les remplacements saisonniers ne remplissent pas l'ensemble des dossiers administratifs.

c) Parmi ces salariés, trois hommes travaillaient comme aide à domicile, ce qui explique l'utilisation du masculin dans ce texte.

d) Certaines interventions ayant lieu le week-end, les salariés interviennent de manière alternée sur certains créneaux horaires. De ce fait, les emplois du temps sont programmés sur deux semaines.

$\left({ }^{*}\right)$ : Ces plans d'aide correspondent à ceux pris en charge par l'association, en janvier 2009.

En effet, le secteur de l'aide à domicile souffre d'un très fort turnover (ANACT, 2009) et de conditions d'emploi parmi les plus mauvaises du point de vue des rémunérations, de la sécurité de l'emploi, des conditions de travail et de la reconnaissance individuelle (Angeloff, 2000 ; Devetter et al., 2009 ; Doniol-Shaw et al., 2007). Les salariés formés au DEAVS restent relativement rares. Entre 2000 et 2009, 49142 personnes ont été diplômées, et cela majoritairement par validation des acquis de l'expérience totale, c'est-à-dire sans aucune formation (Nahon, 2011).

A travers l'analyse d'une structure d'aide à domicile et de ses salariés, cet article vise à comprendre l'impact de l'introduction du DEAVS sur l'emploi des aides à domicile. ${ }^{4}$ On reviendra d'abord sur les objectifs et la mise en place de ce diplôme, le cadre, et la façon dont les acteurs se sont approprié ce diplôme. On étudiera ensuite les caractéris-

4. Cet article est issu d'une recherche qui a bénéficié du programme " Qualité de l'aide au domicile des personnes fragiles » de la Drees (Direction de la recherche, des études, de l'évaluation et des statistiques) et d'une bourse de thèse de la Cnaf (Caisse nationale d'allocations familiales). 
tiques de l'emploi en comparant les conditions d'emploi des différents personnels de l'association étudiée selon leur niveau de diplôme, avant de conclure sur la persistance de la norme de flexibilité.

\section{DEAVS : diplôme clef de voûte de la professionnalisation}

Cette partie positionne le DEAVS dans son environnement, le métier de l'aide à domicile. Elle présente le diplôme et ses modes d'acquisition, son impact sur le financement et la hiérarchisation des tâches ; pour conclure, elle explique comment salariés et employeurs se l’approprient.

\subsection{Objectif théorique, contenu et mode d'acquisition du diplôme}

La mise en place du DEAVS a permis une plus grande visibilité, et donc une plus grande attractivité des emplois d'auxiliaire de vie, en même temps que la construction d'un métier, défini par des frontières pas toujours nettes.

Pour reprendre les propos de Sylvie Moreau, le groupe de travail chargé de la rénovation du certificat d'aptitude aux fonctions d'aide à domicile (CAFAD) et donc de l'aboutissement au DEAVS, a retenu : "L'élargissement et la simplification de l'entrée en formation " (Moreau, 2003, p. 154), les " allègements et dispenses d'épreuves [qui] renforcent l'attractivité du diplôme et son adaptation aux cursus individuels antérieurs et [qui] permettent d'en diminuer les durées de formation et donc les coûts de manière importante". De plus "la procédure de VAE (valorisation des acquis de l'expérience) qui vise à concilier simplicité, fiabilité et lisibilité pour le candidat ", le dispense de certains modules de formation.

En effet, il existe deux formes principales d'obtention du DEAVS : d'une part, la formation continue ou initiale ; d'autre part, la Validation des Acquis de l'Expérience (VAE). "La part des diplômes obtenus par validation des acquis de l'expérience (VAE) [...] dépasse $55 \%$ chez les AVS [auxiliaires de vie sociale] pour lesquels la VAE a été accessible dès 2002. En 2009, 4039 diplômes d'auxiliaire de vie sociale ont été délivrés par VAE (3 799 DEAVS par voie de VAE totale et 240 après une formation complémentaire, c'està-dire par parcours mixte)" (Nahon, 2011).

Quelles que soient les modalités de certification du DEAVS, elles accordent une place très importante aux employeurs. Que ce soit par le biais des stages ou dans le cadre de l'activité professionnelle, support de la VAE, le rôle de l'employeur est essentiel. Ainsi, les employeurs participent directement ou indirectement à la formalisation du contenu des formations (Hatano-Chalvidan, 2012). Alors que la formation visait la construction d'un groupe homogène de professionnels, elle a largement tendance aujourd'hui à 
s'adapter à l'individu, à ses acquis et son expérience (ibid.). Cette nouvelle organisation "permet la construction de parcours individualisés de formation conduisant à une validation ou à une certification" (Bodin-Hullin, Noël, 2006).

Cette individualisation des parcours et la VAE permettent en outre de réduire l'enjeu économique lié à la formation. En effet, la VAE permet de réduire les temps d'absence des salariés de leurs postes de travail. Le corollaire de cette réduction des absences est la réduction des temps de formation à proprement parler. Dans le cadre du DEAVS, cette baisse des temps de formation rend presque impossible la construction d'un espace collectif (Ibid). En effet, dans près de $50 \%$ des cas, ce diplôme est validé uniquement en VAE, c'est-à-dire sans temps de formation et donc sans espace collectif d'apprentissage.

Pour les salariés déjà en emploi et justifiant d'une expérience dans le secteur de l'aide à domicile, l'accès au diplôme est facilité, puisque le DEAVS est majoritairement obtenu par la VAE (Marquier, 2008). Pour Sylvain Ville et Sabrina Nouiri-Mangold (2014), le DEAVS est l'occasion, pour ces candidates, "d'officialiser des savoirs qu'elles ont le sentiment de maîtriser par l'expérience». Ils notent que l'oral de l'examen est l'occasion d'" évaluer la professionnalisation d'un candidat en lui demandant d'expliquer une pratique qu'il a intériorisée ». Le DEAVS viendrait donc valider des pratiques standards ou conventionnelles, même si ces dernières ne correspondent pas à la réalité du travail qui déborde très largement le périmètre légal de l'activité (Bonnet, 2006), trop limité pour garantir la continuité du soin. En effet, si certains actes relèvent du soin et sont donc sanctuarisés pour les professions médicales, nombreux sont les exemples d'aides à domicile clampant des perfusions, refaisant les pansements, coupant les ongles alors que ces tâches leur sont officiellement interdites. L'examen valide ce que "devrait être " l'aide à domicile, c'est-à-dire un accompagnement et non une prise en charge qui souvent leur impose des dépassements de compétences. "Les épreuves du DEAVS en VAE ne sont pas un simple passage d'examen. Comme l'affirment les candidates: "On change (...) on est plus la même", "'est pas la manière de faire qui change, c'est la façon d'être"» (Ibid.).

La formation initiale, quant à elle, repose sur une ambition de connaissance très limitée qui aboutit à des formations empruntant aux compétences dites "féminines " et essayant de les dénaturaliser. Cette dénaturalisation passe par la technicisation d'activités du quotidien, réalisées le plus souvent par des femmes, comme la réalisation des tâches domestiques. La faible institutionnalisation des formations, soulignée par Emilien Julliard et Aude Leroy, (2014), est caractérisée par leur importante variabilité : selon les parcours des formateurs et aussi selon les possibilités de valorisation scolaire. Dans le processus de formation décrit par les auteurs, on retrouve la mise en place d'un modèle industriel de formation par la décomposition systématique de l'activité. Ils présentent le processus de formation, bien plus comme un outil de sélection du public que comme une réelle plus-value à acquérir. Les auteurs montrent la distance entre un enseignement ultra-théorisé et la réalité de l'activité bien loin des standards 
de formation. La formation devient alors une opportunité de socialisation secondaire pour l'entrée sur le marché du travail (Julliard, Leroy, 2014).

Toutefois, le diplôme et la formation qui y est associée ne sont pas intrinsèquement responsables de la hiérarchisation des tâches qui, nous le verrons, affecte la qualité de l'emploi des aides à domicile. En effet, le phénomène est amplifié par les modalités de financement des aides propres à l'APA.

\subsection{Biais du financement par l'APA, hiérarchisation des tâches}

Le conseil général du département étudié finance plus de $55 \%$ de l'activité de l'association et sa grille tarifaire révèle la hiérarchisation des tâches. En 2009, dans le cadre de l'APA, les tarifs horaires appliqués variaient de 20,9€ pour des " aides à la personne " à 19,6€ pour le " ménage ". Cette différenciation n'est pas nationale, en dehors d'un tarif minimum légal, les conseils généraux fixent librement leurs tarifs et peuvent ou non les différencier.

Indépendamment de la différence de financement, ces activités, classées en deux groupes, placent tantôt l'auxiliaire de vie sociale en position d'expert travaillant sur le corps, tantôt en position de salarié subalterne réalisant " des ménages ". La valorisation apportée par le diplôme influence ici la posture du salarié. Se positionnant dans la hiérarchie du soin, l'auxiliaire de vie sociale se valorise et utilise son diplôme pour se différencier des autres aides à domicile non ou moins diplômés, auxquels il laisse donc les activités subalternes, comme le ménage, le linge, l'entretien du cadre de vie.

Notre observation rejoint les travaux d'Anne-Marie Arborio, avec «l'hypothèse sous-jacente à ce raisonnement qui est qu'il existe une hiérarchie des tâches selon leur degré de prestige, de celles qu'on est fier d'exécuter jusqu'aux “corvées" "(Arborio, 1995, p. 108). Ici encore, "passer du travail matériel au travail sur matériau humain constitue donc une promotion" (Ibid., p. 103), matérialisée par un diplôme.

Quels que soient les débats sur le contenu du DEAVS, ce diplôme a eu pour effet de scinder la population des aides à domicile entre ceux qui ont le DEAVS et ceux qui ne l'ont pas. Lors des entretiens avec des salariés diplômés, il ressort à plusieurs reprises que ceux qui ne possèdent pas « le » diplôme ne devraient pas réaliser de soins du corps. Plus tard, lors des discussions avec les gestionnaires de planning, celles-ci précisent que certaines " des filles ", une fois le DEAVS en poche, refusent de réaliser des interventions de " ménage ». Bien que la hiérarchisation des tâches ne soit pas explicite dans le discours des salariés, on note une différenciation entre les salariés formés et les autres, comme l'illustrent les propos d'une auxiliaire de vie évoquant son activité au domicile des personnes âgées : "Bon moi, c'est surtout des toilettes hein, mais la fille qui fait le ménage, parce qu'on est en binôme..." (Femme, 53 ans, mariée, 4 enfants, formée en formation continue au Cafad, avant le recrutement). En effet, plusieurs salariés peuvent intervenir pour différentes tâches au sein d'un même domicile. La différenciation entre les activités est importante aux yeux des salariés formés, comme nous le précise cette autre auxiliaire de vie 
parlant d'intervention différenciée: "Quand on intervient chez une personne, si vous voulez, vu qu'on est à deux et tout, parce que bon ily a aussi bien une aide à la toilette que, admettons, l'entretien de logement" (Femme, 42 ans, mariée, un enfant, formée en formation continue par VAE au DEAVS, après le recrutement). Outre les deux groupes d'activités, les salariés sont conscients de la hiérarchie des tâches : "Donc auxiliaire de vie sociale, c'est aussi bien une aide à la toilette qu'une aide au petit déjeuner parce que c'est toujours le mot "aide" ", en opposition à l'entretien du logement, activité moins spécifique aux personnes vulnérables.

Soulignons que les AVS ne refusent pas catégoriquement de réaliser les activités ménagères lorsqu'elles s'intègrent dans une prise en charge plus importante. En revanche, lorsque les soins se limitent à de l'entretien du logement ${ }^{5}$, ils mettent en avant leur diplôme pour aiguiller ce client vers un collègue non diplômé. Si certains AVS acceptent de réaliser des tâches de ménage auprès des clients, l'acceptation sera d'autant plus facile que la personne sera dépendante. L'activité «ménage » représente de $10 \%$ du temps imparti dans les plans d'aide APA pris en charge par l'association pour les personnes les plus dépendantes, contre $70 \%$ pour les personnes qui le sont peu.

\subsection{Appropriation par les salariés et l'employeur}

Le diplôme n'est pas uniquement un marqueur permettant la hiérarchisation des tâches. En effet, selon son mode d'acquisition, soit il permet aux futurs salariés d'accéder aux employeurs par l'intermédiaire des stages, soit aux employeurs de valoriser symboliquement et financièrement les salariés dévoués.

\subsubsection{Outil de réorientation, importance de l'expérience}

Pour Émilien Julliard et Aude Leroy, l'enjeu de la formation initiale au métier d'aide à domicile est de "convertir des savoir-faire acquis antérieurement par la socialisation familiale aux travaux domestiques en qualités procédurales " (Julliard, Leroy, 2014). Le diplôme permettrait de formaliser un savoir de toute façon déjà assimilé. Dans notre enquête, les CV des salariés ayant validé le DEAVS dans le cadre de la formation continue ou initiale sans expérience professionnelle antérieure, valorisent les stages obligatoires inhérents à la formation. Ainsi, bien que les salariés soient diplômés, l'expérience est toujours mise en avant. La formation apparaît comme un processus d'homogénéisation des pratiques domestiques avec la mise en place de standards.

Le profil des salariés de la première génération de recrutement (avant 2005) s’apparente à la situation, décrite par Olivier Chardon, où "Pabsence de diplôme ou d'expérience professionnelle dans la filière n'est, le plus souvent, pas pénalisante. Les qualifications requises peuvent sobtenir en dehors de la formation initiale " (Chardon, 2005). On note une grande variabilité des formations et des diplômes. Ainsi, en conformité avec les résultats de Christelle

5. Terme utilisé dans les plans d'aide APA du conseil général. 
Avril (2012), beaucoup de salariés enquêtés possédaient un diplôme de niveau BEP/CAP (49/72) dans des domaines comme la comptabilité/administration (17), la couture (6), employé de collectivité (5), le commerce (4), l'horticulture (3), la restauration (3), etc. Six ont un diplôme de niveau bac professionnel, douze de niveau bac général et technique, et cinq du niveau bac +2 .

A première vue, les formations au DEAVS permettraient donc une réorientation des salariés vers l'aide à domicile. Dans la structure étudiée, c'est le cas de 32 salariés. Recrutés principalement après 2005, ils sont déjà diplômés ${ }^{6}$ au moment d'intégrer l'association. Ils ont réalisé plusieurs stages et intègrent l'association dans le cadre de leur premier emploi.

Le recrutement de personnels diplômés du secteur s'amplifie au sein de l'association ${ }^{7}$, mais on constate que ces diplômes se superposent à l'expérience ou en sont les pourvoyeurs. Le diplôme semble souvent couronner un parcours expérimenté ou offrir des possibilités de stage, permettant la réelle professionnalisation. De 1999 à 2005, 44 \% (20/46) des salariés recrutés possèdent des expériences professionnelles dans le secteur de l'aide à domicile. A partir de 2005, et surtout de 2007, la part des salariés recrutés déclarant dans leur CV une expérience professionnelle dans le secteur des services domestiques ou dans une structure médico-sociale passe à $67 \%$ (26/39) sur 2005-2006, puis $72 \%$ (62/86) sur 2007-2009.

Le DEAVS apparaît comme une passerelle vers le métier d'aide à domicile. La formation peut permettre, par son contenu, d'accompagner une réorientation ; néanmoins, il semble que l'une des plus-values du DEAVS soit la possibilité d'acquérir de l'expérience par les stages et par la même d'entrer en relation avec les employeurs.

\subsubsection{La reconnaissance de l'employeur envers le personnel dévoué}

La disponibilité temporelle et la tolérance vis-à-vis des publics difficiles semblent être particulièrement déterminantes dans la définition d'un bon salarié. Elles favorisent la longévité de la relation de travail et donc l'obtention du DEAVS en VAE. Le diplôme correspond à une gratification économique ${ }^{8}$ pour service rendu, et à une certaine reconnaissance

6. Ici, on considère toutes les formations conduisant à l'aide à domicile : DEAVS, CAFAD (Certificat d'aptitude aux fonctions d'aide à domicile), BEP (brevet d'études professionnelles) carièrre sanitaire et sociale ; Diplôme d'Etat d'aide-soignant, BEPA (brevet d'études professionnelles agricoles) option services, spécialité services aux personnes; baccalauréat technologique : série sciences médico-sociales ; titre assistant de vie du ministère du Travail ; titre employé familial polyvalent.

7. Les diplômes de catégorie C (la plus élevée) sont au nombre de 4/46 (9\%) pour les salariés recrutés avant 2005, puis 6/39 (15\%) entre 2005-2006, puis 23/86 (27\%) en 2007-2008.

8. Les salariés titulaires du DEAVS bénéficient d'une rémunération supérieure à leurs collègues. D’après la convention collective, le salaire brut à temps plein l'année de l'embauche sera de $1569 €$ pour la catégorie $\mathrm{C}$ (titulaires du DEAVS) et de $1445,38 €$ pour les catégories A et $\mathrm{B}$, puisque le salaire conventionnel est inférieur au SMIC (salaire minimum interprofessionnel de croissance). Après dix ans de carrière, les salaires mensuels bruts seraient de $1766 €$ pour un temps plein de catégorie C, $1522 €$ pour un temps plein de 
qui, si elle existe, reste principalement symbolique (Bureau, Tuchszirer, 2010). Comme le constate Rémy Marquier (2008), "Les perspectives d'évolution dues à la VAE restent mitigées à court terme : $84 \%$ des candidats précédemment en emploi et ayant obtenu leur diplôme par VAE n'ont changé ni de poste, ni d'employeur", et lorsqu'il y a amélioration, "le changement de situation des candidats ayant obtenu le DEAVS est par ailleurs plus souvent du fait de l'employeur que de l'employé (dans 61 \% des cas)" (Ibid.). Dans le cadre de la VAE, il apparaît d'ailleurs que, dans $77 \%$ des cas, l'initiative de la formation relève de l'employeur (Ibid.).

Notre enquête confirme cette situation. Dans l'association étudiée, nous avons identifié un groupe de salariées formées en formation continue ou en VAE, alors qu'elles travaillent déjà pour l'association. Nous avons choisi d'appeler ce groupe, les "salariées méritantes ». Il compte sept femmes, recrutées en 1999, 2000, 2002, 2003, 2005. Elles sont mariées ou en couple pour six d'entre elles. Nous avons mené un entretien avec deux d'entre elles : "Perle " et "Duchesse ". À la question de savoir si elle était très demandée, "Perle " répond : "Ah oui, très! Et puis, je fais beaucoup les remplacements, jamais je refuse, alors...". De la même manière, "Duchesse " réalise beaucoup de remplacements et sait s'accommoder de personnes difficiles.

Lors de l'observation menée au sein des " bureaux ${ }^{10}$ ", nous avons assisté à des négociations téléphoniques de modifications d'emploi du temps dues à des remplacements, qui facilitaient ou non le travail d'organisation et de gestion du personnel administratif plus proche de la direction. La propension à accepter des cas lourds est aussi valorisée. « Perle » accepte d'intervenir auprès d'une personne " très agressive, vous allez y aller vous ne savez pas comment elle va vous recevoir. D'ailleurs, il y a plein de filles qui y sont allées, elles ne sont pas restées, il faut avoir une sacrée patience!". Alors que de nombreux salariés ont abandonné les interventions auprès de cette cliente, "Perle " continue à intervenir.

Comme nous venons de le démontrer, la volonté d'allègements et de simplification de l'entrée en formation, la procédure de VAE pour l'acquisition du DEAVS se traduisent principalement par des parcours très individualisés au contenu parfois éloigné de la réalité du travail. La formation représente tantôt une opportunité pour le salarié d'entrer en contact avec des employeurs, tantôt pour l'employeur de valoriser certains salariés. Toutefois, une fois le diplôme obtenu, une hiérarchisation s'opère entre les salariés diplômés ou non, par le biais du type de tâches réalisées ; elle est amplifiée par le financement public.

On peut alors s'interroger sur les difficultés d'organisation du travail avec une spécialisation des salariés dans un contexte de dispersion spatiale des lieux d'activité.

catégorie B et $1479 €$ pour un temps plein de catégorie A.

9.Ces noms sont utilisés par le personnel administratif pour nommer ces deux salariés, deux femmes, mariées, diplômées du DEAVS.

10. Terme utilisé par les salariés pour désigner à la fois les locaux de l'association, mais aussi la direction, les responsables de secteur et le personnel administratif, « ceux des bureaux ». 


\section{L'effet mitigé du DEAVS sur la qualité des emplois}

L'emploi d'aide à domicile est un emploi morcelé, irrégulier, d'amplitude horaire importante, majoritairement à temps partiel, et dont la norme d'emploi serait donc flexible hétéronome (Bouffartigue, Bouteiller, 2003), loin de l'emploi moyen. Afin d'observer les effets des diplômes sur l'emploi, nous avons donc recours aux emplois du temps des salariés de la structure étudiée (encadré 1).

Cette structure associative propose des services sous le mode mandataire $(42,1 \%$ des heures) ou prestataire $(57,9 \%)$. Les prestations réalisées en mode prestataire correspondent à des activités de ménage et d'aide à la personne pour $88 \%$. Si l'on ne peut pas s'assurer que les prestations de ménage sont effectivement réalisées dans le cadre de l'aide au maintien de l'autonomie et donc par des aides à domicile, ces prestations représentent $21 \%$ des activités en mode prestataire, laissant $67 \%$ des heures prestées à destination d'un public fragile. D'ailleurs, sur ces 42897 heures réalisées pour un public fragile, 24170 sont directement réglées par le conseil général qui paie les plans d'aide à la structure réalisant le service.

\subsection{Emploi morcelé, amplitude horaire importante}

La modélisation des emplois du temps des salariés de l'association permet d'observer (figure 1) la répartition des deux principales activités réalisées. L'activité de ménage (en bleu), se répartie de manière équilibrée le matin et l'après-midi ; en revanche, l'activité d'aide à la personne (en rouge) se concentre principalement le matin et le soir. Les figures " catégorie d'emploi vs activité " permettent d'observer effectivement une spécialisation des salariés les plus formés (Catégorie $\mathrm{C}$ : Auxiliaire de vie sociale ; Aide médico-psychologique ; Aide-soignant(e) ; Auxiliaire de puériculture) sur les tâches d'aide à la personne, les moins formés, les catégories A (Agent(e) à domicile ; Agent(e) polyvalent ; Agent(e) d'entretien) se concentrent nettement plus sur l'activité de " ménage ». Les catégories B (Employé(e) à domicile ; Employé(e) d'entretien), quant à elles, sont en position intermédiaire en termes d'activité, tout comme elles le sont en termes de diplôme ${ }^{11}$. On observe ainsi une division du travail qui varie selon le niveau de diplôme.

Cette répartition peut s'expliquer par deux phénomènes : d'une part, une explication gestionnaire, où la tarification différente des heures " ménage " et " aide à la personne " par le conseil général incite à orienter les salariés diplômés, c’est-à-dire plus chers et mieux formés, vers les activités d'" aide à la personne ", mieux rémunérées ; d'autre part, une explication liée à la hiérarchisation, venant du refus plus important des salariés formés de

11. Afin d'intégrer la catégorie B, les salariés doivent posséder un BEP ou CAP dans le champ médico-social ; le DEAVS appartient à la catégorie C. 
réaliser des activités de " ménage " moins bien considérées dans la hiérarchie des tâches et qui sont dévolues aux salariés moins formés.

\section{Figure 1 : répartition des interventions des salariés de l'association selon la catégorie d'emploi et le type d'activité réalisée}

\section{(Rouge : « aide à la personne »; bleu : « ménage »)}

Catégorie C (Auxiliaire de vie sociale ; Aide médico-psychologique ; Aide-soignant(e) ; Auxiliaire de puériculture), 18 salariés et 725 interventions ; Catégorie B (Employé(e) à domicile ; Employé(e) d'entretien), 13 salariés et 318 interventions ; Catégorie A (Agent(e) à domicile ; Agent(e) polyvalent ; Agent(e) d'entretien), 42 salariés et 1454 interventions.

Ensemble

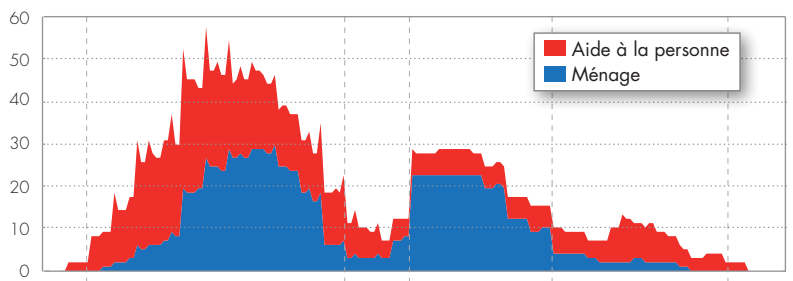

Catégorie A

(les moins

diplômés)

42 salariés et

1454 interventions

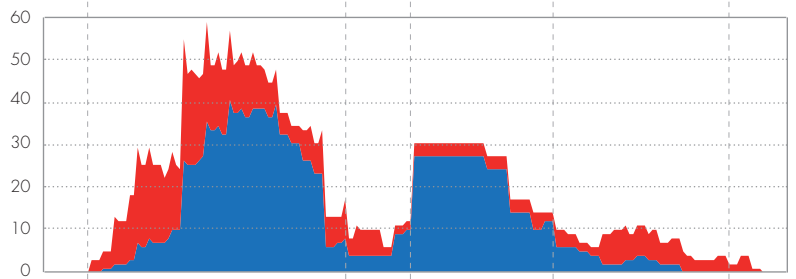

Catégorie B

13 salariés

et 318 interventions
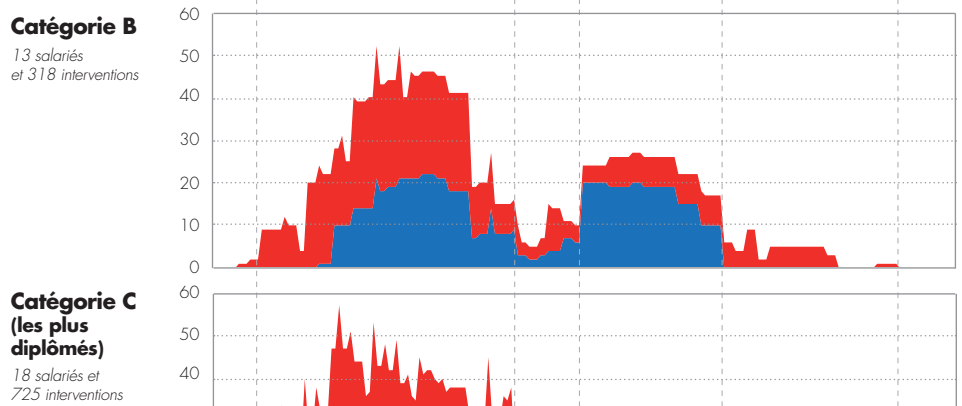

Catégorie C diplômés

725 salaries ef

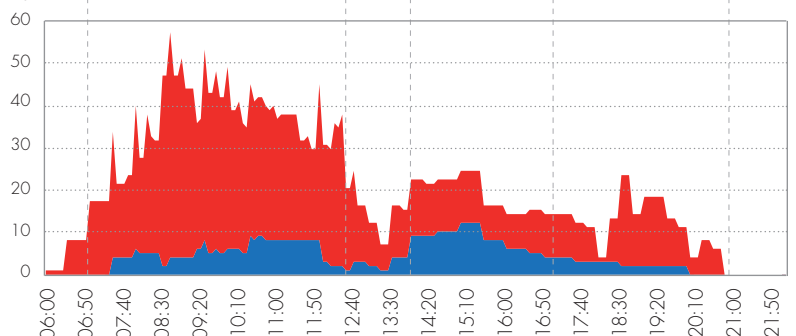

Note de lecture: Pour un salarié moyen, (Ensemble), sa probabilité de travailler entre 14 h et $16 \mathrm{~h}$ est d'environ 0,25, soit 1 jour sur 4 . Cette activité correspond dans $80 \%$ des cas $(0,20 / 0,25)$ à du "ménage " et dans $20 \%(0,05 / 0,25)$ des cas à de l' " aide à la personne ".

Source : emploi du temps sur deux semaines et ramené à un jour, des salariés de l'association dont nous connaissons les caractéristiques de formations. 
La modélisation des emplois du temps témoigne d'une amplitude horaire de l'activité quotidienne nettement plus importante pour les catégories $\mathrm{C}$ (les plus diplômées). Les salariés de cette catégorie travaillent de manière plus fréquente le matin entre $6 \mathrm{~h} 30$ et $8 \mathrm{~h} 00$ et le soir entre $18 \mathrm{~h} 00$ et $21 \mathrm{~h} 00$; en effet, les tâches d'aide à la personne qui leur sont plus fréquemment attribuées en raison de leur formation concernent les individus les plus dépendants. Ce sont en partie des activités d'aide au lever/coucher, à la toilette et à la préparation des repas, souvent associées à un horaire particulier. La spécialisation qui s'opère ici tend donc à accroitre l'amplitude horaire de travail, sans pour autant accroître le temps de travail.

Le temps de travail total pour les deux semaines analysées est ainsi approximativement identique pour les catégories $\mathrm{C}$ et A (48h versus 46h30). Ainsi, il semblerait que l'amplitude plus importante des horaires de travail des salariés diplômés du DEAVS, dans l'association étudiée, ne soit pas compensée par une forte augmentation du temps de travail.

Les résultats de l'enquête GREF Bretagne ${ }^{12}$, interrogeant les salariés formés au DEAVS, CAFAD et CFP assistante de vie deux ans après l'obtention du diplôme, confirment qu'il n'y a pas d'évolution du temps de travail puisque " globalement, le nombre d'heures de travail hebdomadaire n'a pas beaucoup évolué depuis la fin de la formation".

\subsection{Spécialisation des tâches, temps d'intervention}

Notre analyse démontre que le diplôme, en spécialisant ceux qui le détiennent sur les gestes à la personne, raccourcit les temps d'intervention. La comparaison des durées moyennes d'intervention semble désavantager les catégories les plus formées. En effet, alors que la durée moyenne dédiée à chaque intervention, pour l'ensemble des salariés, est de $1 \mathrm{~h} 23$, celle des catégories $\mathrm{C}$ est de $1 \mathrm{~h} 11$. Les salariés en catégorie B réalisent les interventions les plus longues, 1 h37 en moyenne, et les catégories A sont en position intermédiaire, avec $1 \mathrm{~h} 26$.

Pour cette association, la durée des interventions est, à l'exception des prestations de " ménage " simple, majoritairement dictée par l'APA ; celle-ci finance plus de $55 \%$ de l'activité horaire en prescrivant le temps et la fréquence des interventions sous forme d'enveloppe fixe par niveau de dépendance.

Les interventions associées à l'aide à la personne correspondent à des temps d'intervention plus courts et plus souvent quotidiens, alors que le ménage peut être prescrit sur un temps plus long de manière hebdomadaire.

12. GREF Bretagne, Mission Observatoire emploi-formation, insertion professionnelle des personnes ayant obtenu un DEAVS, un CAFAD ou un CFP (certificat professionnel) assitante de vie en 2003, Enquête IROISE, 2006. 
Les règles dictées par l'APA contribuent à morceler l'activité des salariés de catégorie C, les diplômés, et à attirer ces mêmes diplômés vers les tâches les mieux payées, les activités d'aide à temps d'intervention réduit. Ces éléments révèlent qu'à temps de travail équivalent, les salariés les plus diplômés sont ceux qui vont subir le plus grand morcellement de leur activité.

\subsection{Public fragile, contrat fragile}

Les salariés diplômés travaillent surtout pour une population plus dépendante, plus fragile physiquement et par conséquent susceptible d'interrompre le service pour entrer en institution, pour cause d'hospitalisation ou de décès, d'où un risque important de rupture de contrat.

Globalement, les salariés de l'association réalisent $24966^{13}$ interventions programmées dans leurs emplois du temps bi-hebdomadaire ; $23 \%$ sont réalisées dans le cadre d'une contractualisation en CDD (contrat à durée déterminée) et les autres en CDI (contrat à durée indéterminée). Cette proportion apparaît alors affectée par la catégorie du salarié. Ainsi les salariés de catégorie B, c'est-à-dire les salariés formés sans pour autant être mieux rémunérés, interviennent plus souvent dans le cadre d'un contrat CDI (9\% d'interventions en CDD). A l'opposé, les salariés de catégorie C, titulaires du DEAVS ou d'un diplôme équivalent, interviennent moins fréquemment en CDI (34 \% d'interventions en CDD), les salariés des catégories A (les moins diplômés) étant dans une situation intermédiaire avec $21 \%$ d'interventions sous contrat CDD.

On peut faire l'hypothèse ici que les caractéristiques des prestations servies affectent l'emploi dans sa contractualisation. En effet, l'activité d'aide à la personne est soumise à de nombreux aléas, plus rares pour l'activité de ménage. Les deux prestations sont fournies à des publics pouvant être dépendants ; cependant, l'activité d'aide à la personne est orientée spécifiquement vers ce public dépendant, pour lequel la demande est plus instable en raison de problèmes de santé plus importants, de probables hospitalisations ou décès. En revanche, l'activité de ménage s'adresse à une population en meilleure santé et dont l'espérance de vie est potentiellement plus élevée. La stabilisation de l'activité serait alors plus longue et complexe pour les salariés principalement affectés auprès d'un public plus dépendant.

13. 796 interventions programmées sont réalisées par 37 salariés pour lesquels nous ne disposons pas des données relatives à leur formation. La comparaison des rapports 796/37=21,5 et 2496/75=33,3 laisse penser que les salariés pour lesquels certaines informations sont manquantes correspondent à des salariés recrutés plus récemment. 


\subsection{Le recrutement dépend plus de la flexibilité que du diplôme}

Ainsi, nos résultats révèlent que la qualité de l'emploi, au sens de l'organisation du travail et des difficultés liées à l'importante segmentation de l'activité, n'est pas meilleure pour les salariés les mieux formés. Quelle perception les employeurs ont-ils de cette nouvelle élite de l'aide à domicile?

En revenant sur le récent processus de recrutement, bien qu'une amélioration de la professionnalisation et de la qualification des salariés soit constatée, on observe que le critère dominant dans le recrutement demeure la flexibilité du candidat en termes d'emploi du temps et de public, indépendamment de sa formation.

\subsubsection{Exigence de flexibilité en termes de publics et de tâches}

Le graphique 2 retrace l'évolution de la réponse apportée par les salariés à la question sur les publics cibles lors de l'entretien d'embauche. Pendant l'entrevue, le candidat doit remplir un formulaire, avec la question : "Accepteriez-vous de travailler avec les personnes : âgées/handicapées/personnes en activitélenfants ?" En observant les réponses données au cours des dix dernières années, on remarque que les employés recrutés sont de plus en plus ouverts en termes de choix des publics pour lesquels ils acceptent de travailler, mais aussi, de manière sous-jacente, en termes de tâches, et ce, indépendamment de leur formation.

Accepter de travailler pour une " personne en activité ", c'est accepter un contrat de femme de ménage. Que le salarié soit diplômé ou non, on constate que la tendance est la même et que les salariés acceptant de travailler pour tous les publics sont les plus recherchés. Parmi les salariés de catégorie B et C recrutés avant $2005^{14}$, au moment du recrutement, un seul déclare accepter de travailler pour les quatre publics (1/11), cinq pour trois publics, quatre pour deux publics différents et un pour des personnes âgées uniquement. En 2008, sur les douze salariés recrutés, trois déclarent accepter de travailler pour les quatre publics, six pour trois des publics (personnes âgées, personnes handicapées, enfants), et trois pour uniquement deux publics (personnes âgées et handicapées). Finalement, en 2009, sur les six recrutements de salariés de niveau $\mathrm{V}$, tous accepteront de travailler pour les quatre publics.

Malgré une formation des salariés dédiée à un public cible vulnérable, le recrutement favorise ceux qui acceptent de réaliser toutes les activités, dont le ménage. Cette recherche de personnel flexible en termes de tâches et de publics correspond bien plus à la réalité du secteur que la recherche de diplômés. Les emplois du temps révèlent que seul le cumul des différentes activités permet aux salariés de trouver une norme d'emploi plus proche de la norme standard (Devetter, Barrois, 2012).

14. Parmi ces dossiers, un n'est pas renseigné pour les types de publics. Nous avons choisi de regrouper les dossiers des salariés recrutés avant 2005 en raison d'effectifs trops faibles sur les années précédentes. 


\section{Graphique 2 : Flexibilité en termes de publics}

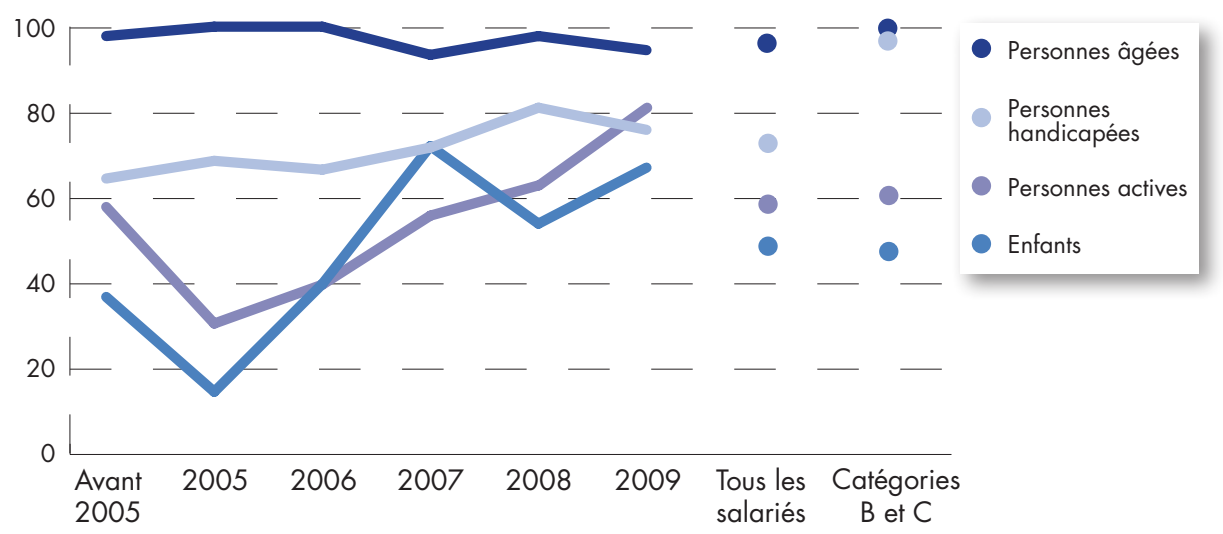

Source: ensemble des données disponibles sur les choix des salariés en termes de public..

Lecture : en 2008, $98 \%$ des salariés recrutés acceptaient de travailler pour aider une personne âgée, $81 \%$ pour aider une personne présentant un handicap, $54 \%$ une personne active et $63 \%$ pour garder des enfants. Globalement, entre 1997 et 2009, l'ensemble des salariés recrutés (151) étaient $72 \%$ à souhaiter travailler pour un public souffrant de handicap contre $97 \%$ des salariés de catégories $B$ et $C(30)$ recrutés sur la même période.

\subsubsection{Exigence de flexibilité en termes d'horaires et de mobilité}

Toutefois, la contrainte de flexibilité ne s'exprime pas uniquement en termes de tâches et de publics. Dans les comptes rendus des entretiens d'embauche réalisés par la maison de l'emploi, il ressort aussi de manière assez précise l'importance de la disponibilité en termes d'amplitude horaire et de mobilité et ce, indépendamment de la formation du salarié. La possession ou non du permis de conduire est un critère présent lors du recrutement, il permet une mobilité du personnel plus importante : en 2008, $76 \%$ des salariés recrutés avaient le permis. Ainsi, des dossiers de candidature sont résumés de cette manière, quel que soit le niveau de formation :

"Madame X est disponible sur une amplitude horaire de 7 à 20h (peut être modifié), la nuit, les week-ends, jours fériés et ok pour la garde à demeure. Elle a le permis et est vébiculée."

Que les salariés soient formés ou non, on constate des caractéristiques similaires s'agissant des publics. En effet, les salariés diplômés du DEAVS se comportent de la même manière que leurs collègues moins diplômés et doivent faire preuve de flexibilité. Ces résultats indiquent que le diplôme n'est qu'un élément utile mais non indispensable lors du recrutement. En outre, ils révèlent que l'attente essentielle des employeurs d'aides à domicile demeure, indépendamment du niveau de diplôme, la flexibilité autant en termes de public, d'horaires, que de tâches, afin de répondre aux contraintes de l'activité. 


\section{Conclusion}

La situation étudiée n'est pas identique sur l'ensemble du territoire en raison de variations autant dans les caractéristiques des acteurs que dans la mise en place des politiques publique ; néanmoins, le processus de segmentation observé entre salariés formés et non formés a une portée générale. En situation de dispersion spatiale de l'activité, il peut aboutir à une taylorisation particulièrement néfaste de l'emploi.

Ainsi, différents éléments plaident pour une analyse nuancée de l'effet du diplôme pour les salariés. L'augmentation du salaire horaire des salariés les plus qualifiés est contrebalancée par la dégradation des conditions d'emploi ; ils sont plus souvent en CDD et interviennent, comme nous l'avons vu, sur des temps plus courts avec une amplitude horaire plus importante du fait d'une segmentation de l'activité séparant " aide à la personne » et " ménage ».

Le processus de différenciation, soutenu par les financements publics, tend à dégrader de manière mécanique la qualité de l'emploi. La hiérarchisation des tâches et la délégation $\mathrm{du}$ "sale boulot ", observables dans l'organisation de nombreuses ${ }^{15}$ structures de soins collectives, n'ont pas la même implication au domicile. En effet, au domicile, la division du travail implique une multiplication des personnels et des interventions engendrant une multitude de déplacements supplémentaires et une parcellisation du travail encore plus importante.

On peut donc partiellement au moins, remettre en question l'objectif ${ }^{16} \mathrm{~d}^{\prime} u n e$ meilleure qualité de l'em,ploi, c'est-à-dire qui tendrait vers un temps complet, avec une amplitude horaire plus faible et une segmentation de l'activité moins importante grâce à la possession du diplôme.

Si effectivement, dans certaines enquêtes, on observe une corrélation entre le DEAVS et l'augmentation du temps de travail (Devetter, Messaoudi, Farvaque, 2012), il semblerait que ceux qui travaillent déjà plus se voient offrir par l'employeur (à l'origine d'une large majorité des demandes de formation (Marquier, 2008)) la possibilité d'obtenir le DEAVS. Bien plus qu'un lien de cause à effet, le DEAVS intervient, dans l'association étudiée, comme un marqueur des salariés les plus " motivés ». En effet, le peu d'impact du DEAVS sur les rémunérations comme sur les perspectives de carrière, et le fait que les demandes émanent des employeurs laissent penser que le DEAVS intervient dans le cadre de la VAE comme une gratification patronale pour les salariés les plus méritants, ceux qui acceptent des horaires flexibles, irréguliers et des publics difficiles.

Les employeurs, incités par les pouvoirs politiques à la diversification de l'activité et des publics, recherchent avant tout, indépendamment du diplôme, des salariés flexibles autant

15. voir notement les travaux de Hughes 1951 ; Arborio, 1995 et Molinier, 2012.

16. Voir le numéro de Formation Emploi 2011/3 (n 115), Dossier : "Le développement de l'aide à la personne : quelle professionnalisation?». 
en termes d'activités que d'horaires. Les salariés diplômés sont pris en étau entre une recherche d'augmentation du temps de travail et une recherche du public cible "vulnérable ${ }^{17}$, pour lequel ils ont été formés.

Indéniablement, la professionnalisation des aides à domicile représente, dans une perspective démographique annonçant une forte croissance du secteur, un objectif sociétal important, autant du point de vue de la qualité du service que de celle des emplois. La mise en place du DEAVS a permis une valorisation de certains salariés situés au bas de l'échelle sociale ; toutefois, elle est impuissante face au morcellement de l'activité qui demeure la plus grande difficulté à résoudre si l'on souhaite réellement améliorer la qualité de l'emploi. Peut-être faut-il alors d'une part, remettre en question les modalités du maintien à domicile et, d'autre part, mieux articuler les choix individuels aux collectifs qui les assument ${ }^{18}$, afin que le maintien de l'autonomie ne se réalise pas au détriment des conditions de travail et d'emploi ${ }^{19}$.

\section{Bibliographie}

Alvarez S. (2010), "Le secteur de l'aide à domicile en France : entre l'imaginaire domestique et l'invisibilité du travail de "care" professionnel ", Communication pour le colloque : La dimension relationnelle des métiers de service : Cache-sexe ou révélateur du genre?, université de Lausanne, septembre.

Anact (2009), Le travail dans les services à la personne, Coll. « Le point sur... », réseau Anact Angeloff T. (2000), Le temps partiel : un marché de dupes?, Paris, Syros, 225 p.

Arborio A.-M. (1995), "Quand le “sale boulot” fait le métier : les aides-soignantes dans le monde professionnalisé de l'hôpital ", Sciences sociales et santé. vol. 13, n 3, pp. 93-126.

Avril C. (2012), "Ressources et lignes de clivage parmi les aides à domicile ", in Actes de la recherche en sciences sociales, $\mathrm{n}^{\circ} 191-192$, mars, pp. 86-105.

17. Pour le code pénal, il s’agit d'une personne dont la particulière vulnérabilité est due à l'âge, à une maladie, à une infirmité, à une déficience physique ou psychique ou à un état de grossesse.

18. Les choix individuels doivent être pensés dans une logique de qualité commune aux personnes aidées et aux salariés. En outre, les structures d'aide ne peuvent apporter une réponse totalement individualisée sans dégrader la qualité du travail et de l'emploi dans une logique de maîtrise budgetaire.

19. On peut ainsi repenser le système afin d'associer les services d'aide à domicile aux services de soins infirmiers à domicile (SSIAD), de limiter la liberté du donneur d'ordre (qu'il soit particulier ou administratif) pour que le prestataire reprenne, dans une certaine mesure, le contrôle de l'organisation de son activité. Un système de lit, comme on peut l'observer avec les SSIAD (Service de soins infirmiers à domicile), et une rémunération directe du prestataire au travers d'un GIR moyen pondéré (indicateur synthétique de besoin moyen en soins de base par individu), comme c'est le cas des maisons de retraite, pourraient permettre de mieux concilier qualité du service et qualité des emplois. 
Bodin-Hullin T., Noël X. (2006), " Partager le langage de la modularisation ", in Trait d'Union, $\mathrm{n}^{\circ}$ 190, décembre, pp. 4-5.

Bonnet M. (2006), « Le métier de l'aide à domicile : travail invisible et professionnalisation ", Nouvelle revue de psychosociologie, $\mathrm{n}^{\circ}$ 1, p.p 73-85.

Bouffartigue P., Bouteiller J. (2003), «A propos des normes du temps de travail. De l'érosion de la norme fordienne aux normes émergentes ", Revue de l'IRES, n 2.

Bureau M.-C., Tuchszirer C. (2010), « La validation des acquis de l'expérience est-elle un moyen de reconnaissance du travail ? ", Sociologie du travail, 52, pp. 55-70.

Chardon O. (2005), « La spécialité de formation joue un rôle secondaire pour accéder à la plupart des métiers ", Économie et Statistiques, n 388-389.

Croff B. \& Mauduit M. (2003), «Travailler auprès de personnes âgées . Une chance pour repartir dans la bonne direction ", Gérontologie et société, vol. 1, n 104, pp. 231-247.

Devetter F.-X. et Barrois A. (2012), «Aides à domicile : un régime temporel non stabilisé qui témoigne des ambiguïtés d'une professionnalisation inachevée. ", Les Conditions de travail des aides à domicile en 2008, Dossiers Solidarité \& Santé, n 30, Drees.

Devetter F.-X., Jany-Catrice F., Fraisse L., Gardin L., Gounouf M.-F. et Ribault T. (2009), L'aide à domicile face aux services à la personne : mutations, confusions, paradoxes, Rapport pour la DIIESES, $300 \mathrm{p}$.

Devetter F-X., Messaoudi D., Farvaque N. (2012), "Contrainte de temps et pénibilité du travail : les paradoxes de la professionnalisation dans l'aide à domicile ", in Revue française des affaires sociales, $n^{\circ} 2-3$, Avril-Septembre, pp. 245-268.

Doniol-Shaw G., Lada E. et Dussuet A. (2007), Les parcours professionnels des femmes dans les métiers de l'aide à la personne : leviers et freins à la qualification et à la promotion, Rapport de recherche du LATTS, novembre.

Hatano-Chalvidan M. (2012), "L'individualisation des parcours de formation dans le champ du travail social : entre tensions et ambivalences ", Formation Emploi, n ${ }^{\circ} 119$, Juillet-Septembre, pp. 83-100.

Hughes E.C. (1951a), "Studying the nurse's work, American Journal of Nursing”, Vol. 51, May, The American Journal of Nursing Company.

Julliard E., Leroy A. (2014), "Convertir des chômeuses, former des professionnelles ? ", in Weber F., Trabut L., Billaud S., (dir.), Le salaire de la confiance, Ed. Rue d'Ulm.

Marquier R. (2008), " Préparer le diplôme d'État d'auxiliaire de vie sociale par la validation des acquis de l'expérience ", Études et Résultats, n 658, Septembre.

Marquier R., Nahon S. (2012), "Les conditions de travail des aides à domicile ", Dossier Solidarité Santé n 30, Drees, juillet. 
Maruani M. (2011), Travail et emploi des femmes, coll. « Repère », La Découverte, $\mathrm{n}^{\circ} 287$.

Mission Observatoire emploi-formation (2006), Insertion professionnelle des personnes ayant optenu un DEAVS, un CAFAD ou un CFP assitante de vie en 2003, Enquête IROISE, GREF Bretagne.

Molinier P. (2012), Le travail de care, Paris, La dispute, coll. " Le genre du monde », 228 p.

Moreau S. (2003), « Du CAFAD au diplôme d'Etat d'Auxiliaire de vie sociale », Gérontologie et Société, n 104, pp. 149-160.

Nahon S (2011), «Les étudiants en formation sociale ou de santé pour l'accompagnement des personnes en perte d'autonomie ", Etudes et résultats, $n^{\circ} 781$, Novembre.

Nahon S (2013), La formation aux professions sociales en 2011, Document de travail, série statistiques $\mathrm{n}^{\circ} 175$, Drees, Janvier.

Nouiri-Mangold S. et Ville S. (2014), "Le poids de l'expérience ", in Weber F., Trabut L., Billaud S., Le salaire de la confiance, Edition Rue d'Ulm, Paris. 